\title{
Acute Kidney Injury at Hospital Admission for SARS-CoV-2 Infection as a Marker of Poor Prognosis: Clinical Implications for Triage Risk Stratification
}

\author{
Carlo Andrea Bravi ${ }^{\mathrm{a}}$ Walter Cazzaniga ${ }^{\mathrm{a}} \quad$ Marco Simonini $^{\mathrm{b}} \quad$ Alessandro Larcher $^{\mathrm{a}}$ \\ Elisabetta Messaggio $^{\mathrm{b}}$ Laura Zagato $^{\mathrm{b}} \quad$ Cristina Carenzi $^{\mathrm{a}}$ Roberto Bertini ${ }^{\mathrm{a}} \quad$ Alberto Briganti $^{\mathrm{a}}$ \\ Paolo Manunta $^{\mathrm{b}}$ Giuseppe Vezzoli $^{\mathrm{b}}$ Andrea Salonia $^{\mathrm{a}}$ Chiara Lanzani $^{\mathrm{b}} \quad$ Umberto Capitanio $^{\mathrm{a}}$ \\ Alberto Zangrillo $^{c}$ Giovanni Landoni ${ }^{c}$ Patrizia Rovere-Querini ${ }^{d, e}$ Moreno Tresoldi ${ }^{f}$ \\ Francesco Montorsi $^{\mathrm{a}}$ Fabio Cicerid, $\mathrm{e}$ \\ aDivision of Oncology/Unit of Urology, Urological Research Institute, URI, IRCCS Ospedale San Raffaele, Milan, Italy; \\ ${ }^{b}$ Nephrology and Dialysis Unit, Genomics of Renal Diseases and Hypertension Unit, IRCCS San Raffaele Scientific \\ Institute, Milan, Italy; 'Department of Anesthesia and Intensive Care, IRCCS San Raffaele Scientific Institute, Faculty \\ of Medicine, Vita-Salute San Raffaele University, Milan, Italy; ${ }^{d}$ Vita-Salute San Raffaele University, Milan, Italy; \\ eDivision of Immunology, Transplantation and Infectious Diseases, IRCCS San Raffaele Scientific Institute, Milan, \\ Italy; ${ }^{\text {DDepartment }}$ of General Medicine and Advanced Care, San Raffaele Scientific Institute, Milan, Italy
}

\section{Keywords}

Coronavirus disease-19. Severe acute respiratory syndrome coronavirus 2 - Urology · Acute kidney injury · Risk stratification · Triage

\begin{abstract}
Background/Aims: The new severe acute respiratory syndrome coronavirus 2 (SARS-CoV-2) causes a wide spectrum of effects, including acute kidney injury (AKI) in up to $40 \%$ of hospitalized patients. Given the established relationship between AKI and poor prognosis, whether AKI might be a prognostic indicator for patients admitted to the hospital for SARS-CoV-2 infection would allow for a straightforward risk stratification of these patients. Methods: We analyzed data of 623 patients admitted to San Raffaele Hospital (Milan, IT) between February 25 and April 19, 2020, for laboratory-confirmed SARS-CoV-2 infection. Incidence of AKI at hospital admission was calculated, with AKI defined according to the KDIGO criteria. Multivariable Cox regression models as-
\end{abstract}

karger@karger.com www.karger.com/kbr

Karger $\stackrel{\text { ' }}{5}$

BOPEN ACCESS
(C) 2022 The Author(s)

Published by S. Karger AG, Basel

This is an Open Access article licensed under the Creative Commons Attribution-NonCommercial-4.0 International License (CC BY-NC) (http://www.karger.com/Services/OpenAccessLicense), applicable to the online version of the article only. Usage and distribution for commercial purposes requires written permission. sessed the association between AKI and overall mortality and admission to the intensive care unit (ICU). Results: Overall, $108(17 \%)$ patients had AKI at hospital admission for SARS-CoV-2 infection. After a median follow-up for survivors of 14 days (interquartile range: 8,23 ), 123 patients died, while 84 patients were admitted to the ICU. After adjusting for confounders, patients who had AKI at hospital admission were at increased risk of overall mortality compared to those who did not have AKI (hazards ratio [HR]: $2.00 ; p=0.0004$ ), whereas we did not find evidence of an association between AKI and ICU admission (HR: 0.95; $p=0.9$ ). Conclusions: These data suggest that AKI might be an indicator of poor prognosis for patients with SARS-CoV-2 infection, and as such, given its readily availability, it might be used to improve risk stratification at hospital admission.

(c) 2022 The Author(s).

Published by S. Karger AG, Basel

Carlo Andrea Bravi, Walter Cazzaniga, Francesco Montorsi, and Fabio Ciceri contributed equally to this work.
Correspondence to:

Carlo Andrea Bravi, bravi.carloandrea@hsr.it 


\section{Introduction}

The new severe acute respiratory syndrome coronavirus 2 (SARS-CoV-2) causes a wide spectrum of effects, ranging from mild, self-limiting respiratory tract illness to severe acute respiratory distress syndrome, multiple organ failure, and death [1-3]. Among various organs and systems targeted by the infection, kidney involvement is a frequent complication, with up to $20 \%$ of patients experiencing acute kidney injury (AKI) during hospitalization for SARS-CoV-2 infection [4]. Overwhelming evidence assessed the role of AKI as a marker of disease severity and survival $[3,4]$, suggesting that early diagnosis of kidney involvement in patients with SARSCoV-2 infection might be crucial to reduce morbidity and mortality. Unfortunately, the role of AKI at hospital admission for SARS-CoV-2 infection has never been investigated. For this reason, we tested the hypothesis that AKI at hospital admission for SARS-CoV-2 infection might be predictive of poor prognosis and as such that it might be used as a triage test for these patients.

\section{Materials and Methods}

We analyzed clinical data of 623 patients admitted to San Raffaele Hospital, Milan (Italy), for laboratory-confirmed SARSCoV-2 infection between February 25 and April 19, 2020 (exclusion criteria are described in online suppl. Fig. 1; for all online suppl. material, see www.karger.com/doi/10.1159/000518271). Demographic characteristics (age and sex), clinical data (comorbidities and outcomes), and laboratory findings during hospitalization were collected from electronic medical records. Patients were stratified according to the presence or absence of AKI at hospital admission, with AKI defined according to the KDIGO criteria [5]. All patients were managed with lopinavir/ritonavir, hydroxychloroquine, and azithromycin as per local institutional standard of care at the time of admission [6]. Supportive therapies with supplemental oxygen and/or noninvasive ventilation with continuous positive airway pressure were provided at the discretion of physicians. Data on baseline renal function (within 1 year prior to hospital admission) were available for approximately $25 \%$ of patients (online suppl. Table 1). For the remaining patients, baseline serum creatinine was estimated using coefficients derived from a multivariable linear regression model predicting baseline renal function for a cohort of 3,319 patients of similar characteristics (online suppl. Table 2). Since controversies exist on the optimal methodology for backestimation of baseline function in acute situations [7], our approach was chosen to ensure a more individualized calculation of baseline serum creatinine.

Our primary goal was to test the hypothesis that AKI at hospital admission for patients with SARS-CoV-2 infection might be predictive of poor prognosis and as such that it might be used as a triage test. Our primary outcome was overall mortality (OM). The secondary outcome was admission to the intensive care unit (ICU).
We first described the characteristics of our cohort, stratified by AKI at hospital admission. Subsequently, the relationship between AKI and each outcome of interest was assessed using multivariable Cox proportional hazards regression models. To adjust for factors that may affect the relationship between AKI and each outcome of interest, our models included the following covariates: age, gender, baseline creatinine, chronic obstructive pulmonary disease, coronary artery disease, and respiratory domain of the sequential organ failure assessment (SOFA) score. When OM was the outcome of interest, we also included admission to the ICU as a time-varying covariate.

\section{Results}

A total of 108 (17\%) patients had AKI at hospital admission for SARS-CoV-2 infection. Demographic characteristics of our cohort are described in online supplementary Table 3 , stratified by AKI. Patients who had AKI at hospital admission were older and had worse baseline creatinine than patients who did not have AKI. Among comorbidities, diabetes, hypertension, chronic obstructive pulmonary disease, and coronary artery disease were more frequent in the AKI group (all $p \leq 0.05$ ).

Overall, 123 patients died after a median follow-up for survivors of 14 days (interquartile range [IQR]: 8, 23). At hospital admission, patients with AKI had a probability of survival at 14 days of $62 \%$ (95\% confidence interval [CI]: $51 \%, 71 \%)$, whereas the predicted overall survival at 14 days for patients without AKI at hospital admission was $90 \%$ (95\% CI: $86 \%, 93 \%$; Fig. 1). A total of 84 patients were admitted to the ICU. Median (IQR) follow-up for patients who were not admitted to the ICU was $12(7,19)$ days. The predicted 7-day probability of freedom from ICU admission for patients with and without AKI when admitted to the hospital was $88 \%$ (95\% CI: 79\%, 93\%) and 90\% (95\% CI: 86\%, 92\%), respectively (online suppl. Fig. 2).

After adjusting for confounders, patients who had AKI at hospital admission were at increased risk of OM compared to those who did not have AKI (hazards ratio [HR]: 2.00; 95\% CI: 1.36, 2.93; $p=0.0004$; Table 1). Although above conventional level of statistical significance, these results were consistent with sensitivity analyses in a subgroup of 146 patients with available data on baseline serum creatinine (HR: 1.94, 95\% CI: 0.98, 3.85; $p=0.059$; online suppl. Table 4). By contrast, we did not find evidence of an association between AKI at hospital admission and the probability of admission to the ICU (HR: 0.95; 95\% CI: $0.51,1.77 ; p=0.9$ ). 


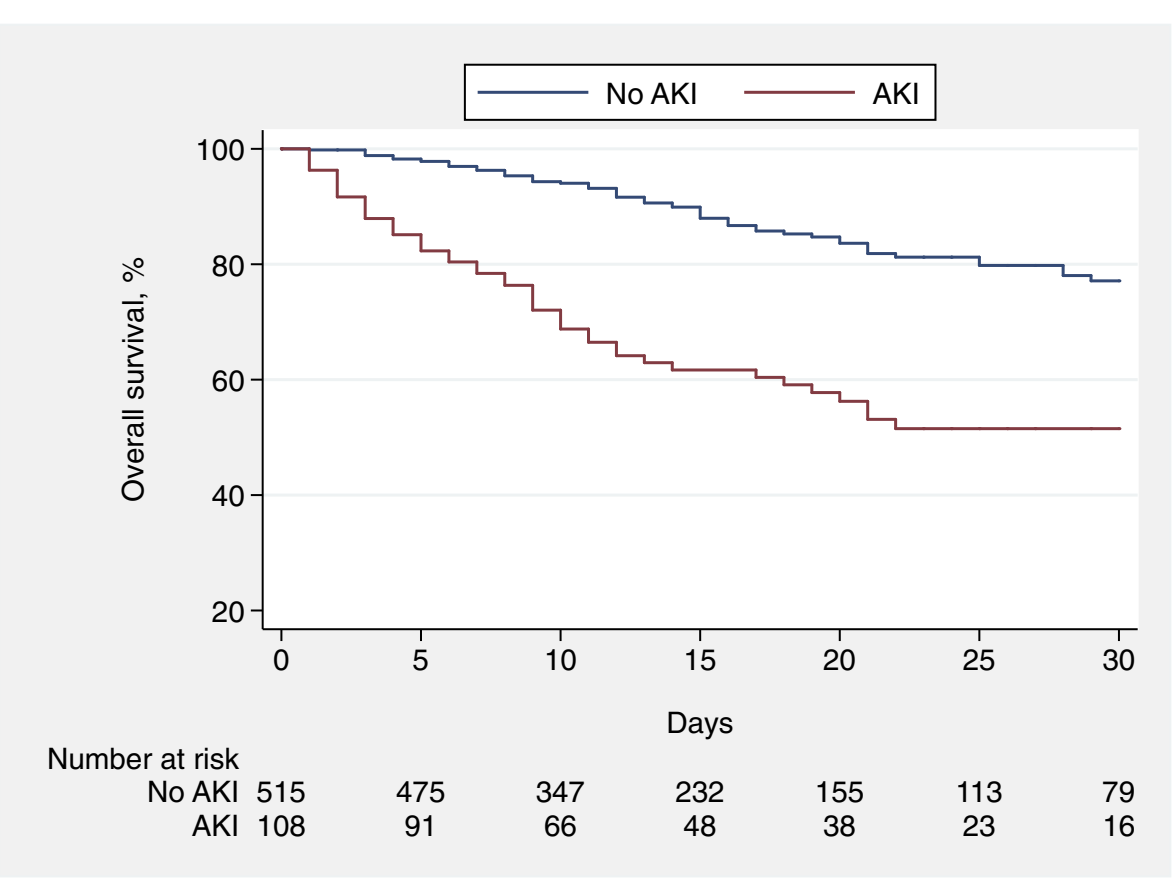

Fig. 1. Overall survival of patients admitted to the hospital for SARS-CoV-2 infection, stratified for AKI at hospital admission. AKI, acute kidney injury.

Table 1. Multivariable Cox regressions assessing the relationship between AKI at hospital admission and overall mortality and admission to the ICU for 623 patients with SARS-CoV-2 infection

\begin{tabular}{llll}
\hline Outcome of interest & $\begin{array}{l}\text { Hazard } \\
\text { ratio }\end{array}$ & $\begin{array}{l}95 \% \\
\text { confidence } \\
\text { interval }\end{array}$ & $p$ value \\
\hline $\begin{array}{l}\text { Overall mortality } \\
\text { AKI at hospital admission } \\
\quad \text { No }\end{array}$ & Ref. & - & \\
$\quad$ Yes & 2.00 & $1.36,2.93$ & 0.0004 \\
$\begin{array}{llll}\text { ICU admission } \\
\text { AKI at hospital admission } \\
\quad \text { No } \\
\quad \text { Yes }\end{array}$ & Ref. & - & \\
\hline
\end{tabular}

Models were adjusted for age, gender, baseline creatinine, COPD (no vs. yes), CAD (no vs. yes), active neoplasia (no vs. yes), and respiratory domain of the SOFA score (categorized as $0-1$ vs. 2 vs. 3-4). When overall mortality was the outcome of interest, the model also included admission to the ICU as a time-varying covariate. AKI, acute kidney injury; COPD, chronic obstructive pulmonary disease; $C A D$, coronary artery disease; ICU, intensive care unit.

\section{Discussion/Conclusions}

We provided key evidence on clinical implications of AKI at hospital admission for SARS-CoV-2 infection, suggesting that given the poor prognosis associated with such construct, AKI might be used as a triage test being a straightforward, easy-to-calculate parameter to stratify patients. This is supported by evidence that although SARS-CoV-2 infection is primarily a respiratory illness, other organs including the kidneys are often affected [2, 8]. Accordingly, it is plausible that patients experiencing a more aggressive, multiorgan onset of SARS-CoV-2 infection might be at higher risk of poor prognosis, as suggested by a recent meta-analysis [9]. Moreover, this is consistent with the relationship between AKI and respiratory failure [10] and cardiovascular events [11], as well as with the detrimental effect of AKI on subsequent recovery of renal function $[12,13]$.

Our study is not devoid of limitations. Data on urine output, a component of the current classification criteria for AKI, were not available for the analyses. Still, we are confident that the definition of AKI using other clinical parameters included in the KDIGO criteria remains sound. Moreover, although our statistical models were adjusted for case mix, we cannot exclude residual confounding from known and unknown variables that may affect the relationship between AKI and outcomes of interest. For instance, data on diuretics or antihypertensive drugs were not available for the analyses. In addition, the exact duration of SARS-CoV-2 infection before hospital admission was unknown. We also have to acknowledge that we analyzed data from the early phase of SARSCoV-2 pandemic, when hospitals experienced a shortage 
of available beds, especially in ICU. This might explain the fact that we did not find evidence of an association between AKI at hospital admission and ICU admission. Finally, patients with available data on baseline creatinine had slightly worse health status than that of patients whose baseline function was estimated. However, the rate of AKI did not differ between the groups (online suppl. Table 4). Moreover, our models were adjusted for both patient characteristics and SARS-CoV-2 respiratory severity, and as such we are confident that this did not affect our results. Despite these limitations, our study represents the first study assessing clinical implications of AKI at hospital admission for SARS-CoV-2 infection, providing relevant insight on the natural history of this disease and thus potentially improving the management of these patients.

In conclusion, we found an association between AKI at hospital admission for SARS-CoV-2 infection and survival. Therefore, our data suggest that such construct might be informative for triage purposes during SARSCoV-2 outbreak.

\section{Statement of Ethics}

All participants have given their written informed consent for data collection as part of the COVID-19 institutional clinical-biological cohort assessing patients with COVID-19 (Covid-BioB, ClinicalTrials.gov NCT04318366) at the Istituto di Ricovero e Cura a Carattere Scientifico (IRCCS) San Raffaele Hospital, Milan, Italy.

\section{Conflict of Interest Statement}

The authors have no conflicts of interest to declare.

\section{Funding Sources}

The authors have no funding sources to acknowledge.

\section{Author Contributions}

Bravi contributed to conceptualization, data curation, formal analysis, roles/writing - original draft, and writing - review and editing. Cazzaniga contributed to conceptualization, data curation, formal analysis, roles/writing - original draft, and writing review and editing. Simonini contributed to interpretation. Larcher contributed to interpretation. Messaggio contributed to data curation. Zagato contributed to data curation. Carenzi contributed to data curation. Bertini contributed to supervision. Briganti contributed to supervision. Manunta contributed to supervision. Vezzoli contributed to supervision. Salonia contributed to supervision. Lanzani contributed to supervision and writing - review and editing. Capitanio contributed to supervision and writing - review and editing. Zangrillo contributed to supervision. Landoni contributed to supervision. Rovere-Querini contributed to supervision. Tresoldi contributed to supervision. Montorsi contributed to supervision. Ciceri contributed to supervision.

\section{Data Availability Statement}

Data is available for review.

\section{References}

1 Grasselli G, Pesenti A, Cecconi M. Critical care utilization for the COVID-19 outbreak in Lombardy, Italy: early experience and forecast during an emergency response. JAMA. 2020;323:1545-6.

2 Rocco C, Reis T, Husain-Syed F. Management of acute kidney injury in patients with COVID-19. Lancet Respir Med. 2020;87:738-42.

3 Zhou F, Yu T, Du R, Fan G, Liu Y, Liu Z, et al. Clinical course and risk factors for mortality of adult inpatients with COVID-19 in Wuhan, China: a retrospective cohort study. Lancet. 2020;395:1054-62.

4 Richardson S, Hirsch JS, Narasimhan M, Crawford JM, McGinn T, Davidson KW, et al. Presenting characteristics, comorbidities, and outcomes among 5700 patients hospitalized with COVID-19 in the New York City area. JAMA. 2020;323:2052-9.
5 Khwaja A. KDIGO clinical practice guidelines for acute kidney injury. Nephron Clin Pract. 2012;120:c179-84.

6 Ciceri F, Castagna A, Rovere-Querini P, De Cobelli F, Ruggeri A, Laura G, et al. Early predictors of clinical outcomes of COVID-19 outbreak in Milan, Italy. Clin Immunol. 2020; 217:108509.

7 Pickering JW, Endre ZH. Back-calculating baseline creatinine with MDRD misclassifies acute kidney injury in the intensive care unit. Clin J Am Soc Nephrol. 2010;5(7):1165-73.

8 Huang C, Wang Y, Li X, PhD PLR, MD PJZ, $\mathrm{MD} \mathrm{YH}$, et al. Clinical features of patients infected with 2019 novel coronavirus in Wuhan, China. Lancet. 2020;395:497-506.
9 Brienza N, Puntillo F, Romagnoli S, Tritapepe L. Acute kidney injury in coronavirus disease 2019 infected patients: a meta-analytic study. Blood Purif. 2021;50:35-41.

10 Hirsch J, Ng J, Ross D, Shah HH, Barnett RL, Hazzan AD, et al. Acute kidney injury in patients hospitalized with COVID-19. Kidney Int. 2020;98:209-18.

11 Legrand M, Rossignol P. Cardiovascular consequences of acute kidney injury. N Engl J Med. 2020;382:2238-47.

12 Bravi CA, Vertosick E, Benfante N, Tin A, Sjoberg D, Hakimi AA, et al. Impact of acute kidney injury and its duration on long-term renal function after partial nephrectomy. Eur Urol. 2019;76:398-403.

13 Bravi CA, Capitanio U. Why acute kidney injury during partial nephrectomy matters. Ann Transl Med. 2020;8:134. 